\title{
Preditores do medo de cair em idosos portugueses na comunidade: um estudo exploratório
}

\author{
Predictors of the fear of falling among community-dwelling \\ elderly Portuguese people: an exploratory study
}

${ }^{1}$ Departamento de Educação e Psicologia, Escola Superior de Saúde, Universidade de Aveiro (ESSUA). Campus Universitário de Santiago Edifício 30, Agras do Crasto. 3810-193 Aveiro Portugal. scas@ua.pt

${ }^{2}$ Departamento de

Educação e Psicologia,

ESSUA. Aveiro Portugal.
Abstract The fear of falling constitutes a real risk factor for falls. It has also been associated with functional decline, decreased quality of life and increased social isolation among the elderly population. This study analyzed predictors of the fear of falling in elderly Portuguese people that live in the community. A cross-sectional study with a convenience sample of 98 participants (57.1\% women; mean age $74.07 \pm 8.74$ years) was conducted. Data were collected with a protocol which includes a questionnaire for sociodemographic and health information, the Activity Specific Balance Confidence Scale, the Hospital Anxiety and Depression Scale, the Lubben Social Network Scale-6, the Timed Up and Go, and Five Times Sit to Stand Test. Data were analyzed using descriptive and inferential statistics. The results showed that female gender $(p=0.01)$, the perception of $\operatorname{good}(p=0.01)$ and moderate $(p=0.02)$ physical health and the symptoms of depression $(p \approx 0.00)$ are predictors of fear of falling. Controlling these predictors is a fundamental aspect for promoting the independence of elderly people minimizing the consequences that are associated with the fear of falling.

Key words Elderly people, Fear of falling, Predictors, Community
Resumo O medo de cair é um fator de risco de queda. Também tem sido associado ao declínio funcional, diminuição da qualidade de vida e aumento do isolamento social na população idosa. Este estudo teve como objetivo analisar preditores do medo de cair em pessoas idosas portuguesas residentes na comunidade. Foi desenvolvido um estudo transversal com uma amostra de conveniência de 98 participantes (57,1\% mulheres; média etária $74,07 \pm 8,74$ anos). O protocolo de coleta de dados incluiu um questionário sociodemográfico $e$ de saúde, a Escala de Confiança no Equilíbrio específica para a Atividade, a Escala de Ansiedade e Depressão Hospitalar, a Escala Breve de Redes Sociais de Lubben, o Timed Up and Go, e o Teste de Sentar e Levantar Cinco Vezes. Os dados foram analisados com recurso à estatística descritiva $e$ inferencial. Os resultados indicam que no gênero feminino ( $p=0.01)$, a percepção de saúde física boa $(p=0.01)$ e moderada $(p=0.02)$ e os sintomas de depressão $(p \approx 0.00)$ são preditores do medo de cair. Controlar estes preditores é um aspecto fundamental para a promoção da independência das pessoas idosas, minimizando as consequências associadas ao medo de cair.

Palavras-chave Pessoas idosas, Medo de cair, Preditores, Comunidade 


\section{Introdução}

O aumento da esperança de vida e da proporção de pessoas idosas nas estruturas demográficas tem suscitado várias preocupações em torno dos episódios incapacitantes, nomeadamente a ocorrência de quedas.

O grupo ProFaNE (Prevention of Falls Network Europe $)^{1}$ definiu a queda como "um evento inesperado no qual o participante desce para o chão ou outro nível inferior".

As quedas são uma importante causa de morbilidade e mortalidade ${ }^{2}$ representando um problema de saúde pública ${ }^{3}$ e uma das grandes síndromes geriátricas ${ }^{4,5}$. Estima-se que todos os anos cerca de 30\% das pessoas idosas sofram, pelo menos, uma queda ${ }^{6,7}$, número que aumenta para $32 \%$ a $42 \%$ em pessoas com mais de 70 anos $^{8}$ e cerca de $50 \%$ para pessoas com mais de 80 anos $^{7}$.

De acordo com a Organização Mundial de Saúde ${ }^{3}$, as pessoas com mais de 70 anos, principalmente do sexo feminino, apresentam taxas de mortalidade associadas a quedas superiores às pessoas mais jovens. As causas para este fenômeno podem estar associadas à diminuição da força muscular ${ }^{9}$, à osteoporose, à prevalência de doenças crônicas e a uma maior restrição à vida doméstica $^{10,11}$.

Tinetti e Kumar ${ }^{12}$ revelam que os fatores de risco mais associados à ocorrência de quedas são a existência de quedas anteriores, a medicação e os distúrbios da força, da marcha e do equilíbrio.

De fato, as quedas têm um impacto significativo na qualidade de vida e saúde da própria pessoa que caiu, na sua família e na comunidade, implicando custos elevados em cuidados de saúde ${ }^{7}$. Estima-se que $70 \%$ das quedas levam ao aparecimento do medo de cair $^{13}$, e que este é observado em cerca de $20,8 \%$ a $85 \%$ da população idosa ${ }^{5}$. Estima-se também que $12 \%$ a $65 \%$ da população idosa a viver na comunidade e que não tenha sofrido qualquer queda apresente medo de cair $^{14,15}$. A prevalência de medo de cair parece aumentar com a idade e ter maior prevalência no sexo feminino ${ }^{16,17}$.

Tinetti e Powell ${ }^{18}$ descreveram o medo de cair como uma "preocupação permanente em relação à queda que leva a pessoa idosa a evitar desempenhar as atividades de vida diárias".

$\mathrm{O}$ medo de cair tem sido reconhecido como um fator de risco de quedas e um determinante de funcionalidade e qualidade de vida na população idosa ${ }^{5,14,19}$. Há evidências que o medo de cair leva à restrição da atividade e participação, aumentando a perda da confiança e diminuindo a capacidade para evitar as quedas ${ }^{13,20}$. Esta restrição da atividade é observada em $50 \%$ das pessoas idosas com medo de cair ${ }^{13}$ e pode originar declínio funcional, atrofia muscular, falta de equilíbrio, alterações na marcha, depressão, isolamento social, com repercussões evidentes na qualidade de vida ${ }^{4,14,15,19}$.

A prevalência do medo de cair nas pessoas idosas representa uma estreita relação com múltiplos fatores, nomeadamente a idade avançada, ser do sexo feminino, reduzida satisfação com a vida, fraca percepção do estado de saúde e de autoeficácia, sedentarismo e obesidade, falta de equilíbrio, decréscimo na mobilidade e marcha, viver sozinho e/ou com rede social reduzida, índices baixos de atividade física, problemas de visão, polimedicação e histórico de queda ${ }^{6,16,17}$.

Na literatura científica o medo de cair é descrito como um fenômeno psicológico que leva a estados de ansiedade e sintomas depressivos e distresse psicológico ${ }^{4,15,19,20}$, o que exige uma maior atenção no controle percebido sobre o cair $^{14,21}$.

Já a inatividade decorrente dos sintomas associados ao medo de cair pode resultar em graves alterações emocionais, psicológicas e sociais, traduzindo-se em perda de autonomia e independência com implicações no desempenho das atividades de vida diária (AVD) das pessoas idosas e a um aumento dos sentimentos de fragilidade e insegurança ${ }^{22,23}$. Por sua vez, o isolamento social, a redução ou mesmo restrição das AVD, os sintomas de depressão, a perda de independência funcional aumenta o medo de cair podendo resultar no aumento do risco de queda ${ }^{5,17,19,20}$ e ser um dos fatores mais incapacitantes no cotidiano das pessoas idosas ${ }^{22}$.

Desconhecem-se estudos em Portugal acerca dos preditores do medo de cair com pessoas idosas a viver na comunidade, assim, este estudo teve como objetivo analisar os preditores do medo de cair em pessoas idosas portuguesas na comunidade.

\section{Metodologia}

\section{Desenho do estudo e participantes}

Foi conduzido um estudo transversal, de caráter descritivo-correlacional. Considerando o medo de cair, avaliado pela Escala de Confiança no Equilíbrio específica para a Atividade ${ }^{24}$ como variável dependente, estabeleceu-se uma amostra total com um mínimo de 80 participantes, de 
acordo com os critérios propostos por Bryman e Cramer ${ }^{25}$, segundo os quais o tamanho da amostra dever ser no mínimo igual a cinco vezes o número de itens da escala. O método de amostragem foi não-probabilístico por conveniência e os critérios de inclusão para participação no estudo foram os seguintes: indivíduos de nacionalidade portuguesa com idade igual ou superior a 60 anos; aceitar participar voluntariamente no estudo; e assinar o termo de consentimento livre e esclarecido. Foram excluídas todas as pessoas: acamadas; hospitalizadas nos últimos 3 meses; dependentes do uso de cadeira de rodas; com amputação das extremidades inferiores; institucionalizadas; e com diagnóstico clínico de perturbação psiquiátrica grave.

\section{Procedimentos de coleta de dados e considerações éticas}

O processo de coleta de dados decorreu entre Dezembro de 2014 a Fevereiro de 2015, após obtenção de parecer favorável pela Comissão de Ética da Unidade de Investigação em Ciências da Saúde: Enfermagem (UICISA: E). As direções de paróquias e centros de convívio na região centro de Portugal foram contatadas presencialmente pela primeira autora. Neste contato, foram explicados os objetivos e metodologias do presente estudo, e solicitada a colaboração para identificar potenciais participantes. Cumpridas as formalidades de pedidos de autorização às entidades identificadas, e após parecer positivo, estas identificaram potenciais participantes que respeitassem os critérios de inclusão/exclusão e mediaram os contatos entre a primeira autora e os participantes. Previamente à coleta de dados, todos os participantes foram esclarecidos quanto à natureza e objetivos do estudo, métodos e meios pelos quais ele seria conduzido, solicitando a sua participação voluntária. Foram identificados 103 participantes, mas 5 recusaram participar do estudo por falta de tempo. Após os esclarecimentos e concordância em participar, os mesmos foram convidados a assinar o termo de consentimento livre e esclarecido.

Para a coleta de dados foi administrado um protocolo por entrevista, aplicado na residência de cada participante e com duração aproximada de 30 minutos que incluiu um questionário de informação sociodemográfica e de saúde, a Escala de Confiança no Equilíbrio específica para a Atividade (CEA) ${ }^{24}$, a Escala de Ansiedade e Depressão Hospitalar (HADS) ${ }^{26}$, a Escala Breve de Redes Sociais de Lubben (LSNS-6) ${ }^{27}$, o Timed Up and Go (TUG) ${ }^{28}$, e o Teste de Sentar e Levantar Cinco Vezes $\left(\text { TSL5 }^{*}\right)^{29}$.

\section{Instrumentos de avaliação}

\section{Questionário de informação sociodemográfica e de saúde}

Este questionário, dividido em duas partes foi desenvolvido com base na checklist da Classificação Internacional de Funcionalidade, Incapacidade e Saúde ${ }^{30}$. A primeira parte é composta por questões que visam recolher informação sociodemográfica (gênero, idade, escolaridade, estado civil, ocupação habitual) e a segunda parte é composta por questões relativas à saúde (quedas, medo de cair, percepção de saúde física, mental e emocional).

\section{Escala de Confiança no Equilíbrio específica para a Atividade (Escala CEA)}

A Escala de Confiança no Equilíbrio específica para a Atividade (Escala CEA) (no original, Activities-specific Balance Confidence Scale-ABC) é uma das mais utilizadas para avaliar o medo de cair. Foi desenvolvida por Powell e Myers (1995) e é usada para medir a confiança na capacidade de manter o equilíbrio durante a realização das $\mathrm{AVD}^{5,21}$. Trata-se de um questionário constituído por 16 itens avaliados numa escala visual analógica, cuja pontuação varia entre $0 \%$ ("sem nenhuma confiança”) e 100\% ("confiança completa”). Quanto maior a pontuação obtida, maior o nível de confiança no desempenho das $16 \mathrm{AVD}^{31}$.

A CEA apresenta, para a população idosa na comunidade, adequada consistência interna com um valor de alpha de Cronbach de $0,96^{32}$ e fiabilidade teste-reteste elevada $(r=0,92 ; \mathrm{p}<0,001)^{31}$. A versão portuguesa deste instrumento demonstrou boa fiabilidade teste-reteste e inter-observador, e adequada consistência interna (alpha de Cronbach de 0,95$)^{24}$.

Escala de Ansiedade e Depressão Hospitalar A Escala de Ansiedade e Depressão Hospitalar (no original, Hospital Anxiety and Depression Scale - HADS) foi desenvolvida por Zigmond e Snaith em 1983, com o objetivo de auxiliar os profissionais de saúde no reconhecimento de componentes emocionais associadas à doença física e que podem afetar os níveis de distresse provocados pela doença, confundir o diagnóstico e prolongar a recuperação ${ }^{26}$. Esta escala permite avaliar a depressão e a ansiedade em contexto hospitalar, no entanto mostrou ser útil em con- 
textos de saúde de cuidados primários, como centros de saúde, centros comunitários, contextos psiquiátricos, assim como um instrumento de rastreio da ansiedade e depressão para a população em geral ${ }^{26}$.

Trata-se de um instrumento de autorresposta composto por duas subescalas: uma que mede a ansiedade (HADS-A) e outra a depressão (HADS -D). Cada subescala tem 7 itens, com 4 opções de resposta numa escala de tipo Likert, pontuadas de 0 a 3, atingindo uma pontuação máxima de 21 pontos cada. As pontuações normativas baseadas no estudo para a validação da versão portuguesa da HADS consideram os seguintes valores para a ansiedade e depressão: 0 a 7 - normal, 8 a 10- leve, 11 a 14 - moderada, e 15 a 21 - severa ${ }^{26}$. Vários estudos recomendam como ponto de corte as pontuações que variam entre $8 / 9$, contudo, este valor não foi estudado para a população portuguesa. No entanto, pode assumir-se, para efeitos de rastreio, estes valores, dada a semelhança das restantes propriedades psicométricas com as versões originais e adaptadas para outros países ${ }^{26}$. Neste estudo foi considerado como ponto de corte a pontuação igual ou superior a 8 que é indicativo de sintomatologia ansiogena/depressiva.

A versão portuguesa da HADS evidencia propriedades psicométricas semelhantes às de estudos internacionais, com valores de alpha de Cronbach de 0,76, para a subescala ansiedade, e de 0,81 , para a subescala depressão, e o valor de correlação entre as escalas de $0,58^{26}$.

\section{Escala Breve de Redes Sociais de Lubben (LSNS-6)}

A Escala Breve de Redes Sociais de Lubben (no original, Lubben Social Network Scale 6 LSNS-6) é um instrumento breve, constituído por seis itens que avaliam o isolamento social em pessoas idosas, através da sua percepção de apoio social recebido por familiares e $\operatorname{amigos}^{27}$. A LSNS-6 tem por base duas subescalas: uma que avalia as relações familiares e outra, as relações de amizade, com três itens cada. A pontuação total resulta do somatório dos seis itens, que varia de 0 a 30 pontos, sendo que as respostas a cada um dos itens variam numa escala entre 0 e 5 . Cada subescala apresenta uma pontuação que varia entre 0 e 15. Para facilitar a interpretação da pontuação total da LSNS-6, foi identificado o ponto de corte de 12 , pelo que a pessoa idosa que apresente pontuações inferiores a 12 é identificada como socialmente isolada ${ }^{27}$.

A escala LSNS-6 apresenta adequada consistência interna com valor de 0,798 , medida através do alpha de Cronbach. As subescalas apresentam igualmente uma consistência interna adequada, com valores de 0,756 e 0,732 para a subescala "família" e subescala "amigos", respectivamente ${ }^{27}$. A análise de correlação item-total (obtida com recurso coeficiente de correlação intraclasse - CCI) assume valores entre 0,457 e 0,631, indicando a homogeneidade dos itens da escala. Por outro lado, as correlações item-subescala assumem valores entre 0,552 e 0,649 para a subescala "Família" e entre 0,466 e 0,657 para a subescala "Amigos"27.

\section{Timed Up and Go (TUG)}

O Timed Up and Go (TUG) foi elaborado por Mathias, Nayak e Isaacs, em 1986, e adaptado por Podsiadlo e Richardson ${ }^{28}$, em 1991, tendo por objetivo avaliar a mobilidade funcional, o risco de queda e os distúrbios da marcha e do equilíbrio. O teste consiste no participante levantar-se de uma cadeira (com altura do assento aproximadamente de $46 \mathrm{~cm}$ e altura de braço de $65 \mathrm{~cm}$ ), sem ajuda dos braços, andar numa distância de três metros, virar-se $180^{\circ}$, voltar em direção à cadeira e sentar-se. O participante deve receber a instrução "vá" para iniciar o teste e o tempo será cronometrado em segundos, a partir da instrução até o momento em que apoie novamente o dorso no encosto da cadeira ${ }^{28,29,33}$.

Através deste teste é possível distinguir pessoas idosas com desempenho normal e independentes nas AVD quando realizam a tarefa em menos de 10 segundos; acima de 30 segundos, os participantes são considerados dependentes na maioria das AVD e na mobilidade ${ }^{28}$. Embora não haja consenso na literatura, sugere-se o ponto de corte do TUG de 12 segundos e as pessoas idosas com tempos mais longos na execução do teste estarão mais propensas a cair ${ }^{34}$. Neste estudo foi considerado o melhor desempenho em duas tentativas.

O TUG tem apresentado boas propriedades psicométricas, nomeadamente em termos de fiabilidade teste-resteste (CCI $=0,75$ e 0.93 ) e inter -observador $(\mathrm{CCI}=0.99)^{33}$.

\section{Teste de Sentar e Levantar Cinco Vezes $\left(\right.$ TSL5 $^{\star}$ )}

O Teste de Sentar e Levantar Cinco Vezes (no original, Five Times Sit to Stand Test - FTSS) é um teste simples, rápido e não invasivo utilizado para avaliar o equilíbrio funcional e a confiança no equilíbrio ${ }^{35}$. Foi desenvolvido e validado em 1985 por Csuka e McCarty e avalia o tempo necessário para uma pessoa se sentar e levantar de uma cadeira cinco vezes consecutivas ${ }^{36}$. 
Este teste inicia-se com o participante sentado com os braços cruzados sobre o peito e com as costas apoiadas no encosto da cadeira. Após garantir que a postura está correta, o pesquisador dá as instruções quero que se levante e sente cinco vezes, o mais rápido que conseguir, assim que eu disser 'vá' e pede ao participante para se levantar na totalidade entre as repetições do teste sem tocar no encosto da cadeira durante as mesmas ${ }^{37}$. O tempo começa a ser cronometrado quando o pesquisador pronuncia a palavra "vá" e termina quando o participante se senta após a quinta repetição $0^{37}$. O tempo que o participante demora para completar o teste é registado em segundos e são realizadas duas tentativas separadas por um minuto de descanso entre elas ${ }^{35}$.

Segundo Bohannon ${ }^{29}$, os valores de referência do teste devem refletir a idade: 11,4 segundos (60 a 69 anos); 12,6 segundos (70 a 79 anos); e 14,8 segundos ( 80 a 89 anos). Neste estudo foi considerado o melhor desempenho em duas tentativas.

O TSL5* tem apresentado boas propriedades psicométricas em termos de fiabilidade teste-reteste para pessoas idosas na comunidade e de validade ${ }^{38,39}$.

\section{Procedimentos de análise de dados}

Os dados relativos à caracterização da amostra foram analisados com recurso à estatística descritiva através do cálculo de frequências absolutas e relativas e de medidas de tendência central e dispersão (média e desvio padrão).

Utilizou-se a regressão linear múltipla para determinar a influência das variáveis independentes (gênero, idade, educação, medo de cair, percepção de saúde física, mental e emocional, ansiedade, depressão, isolamento social, mobilidade e equilíbrio funcional) sobre o comportamento da variável dependente, a pontuação total na Escala CEA. A variável independente permaneceu no modelo se apresentou um nível descritivo do teste menor que 0,05 .

Para a construção dos modelos de regressão linear múltipla as variáveis qualitativas com duas categorias foram codificadas como 0 ou 1 , em que o valor do coeficiente obtido refere-se à categoria da variável codificada como 1 .

Para todas as variáveis analisadas o nível de significância estatística foi definido como $=0.05$ e os dados foram analisados através do programa estatístico Statistical Package for Social Sciences (SPSS), versão 22 para Windows.

\section{Resultados}

\section{Participantes}

A amostra foi constituída por 98 participantes com uma média etária de 74,07 $\pm 8,74$ anos, sendo que $56(57,1 \%)$ são do gênero feminino (Tabela 1). Relativamente às habilitações literárias predomina a escolaridade entre o 1 e 4 anos $(\mathrm{n}=76)$. A maioria dos participantes é casada ( $\mathrm{n}$ $=72)$ e encontra-se na situação de reforma ( $\mathrm{n}=$ 81).

A generalidade dos participantes relatou não ter sofrido qualquer queda no último ano $(\mathrm{n}=$ 80) e o número médio de quedas por pessoa/ano foi de $0,28 \pm 0,64$. No entanto, 48 participantes relataram sentir medo de cair.

A maior parte considera a sua saúde física ( $\mathrm{n}$ = 73) e mental ( $\mathrm{n}=76)$ "moderada" e/ou "boa".

$\mathrm{Na}$ escala CEA observou-se uma pontuação média de 85,93 $\pm 17,29$, sendo que 93 participantes obtiveram uma pontuação igual ou superior a $50 \%$.

Observou-se uma pontuação média de 4,81 $\pm 5,56$ para a subescala de ansiedade (HADS-A), e de $4,87 \pm 5,71$ para a subescala de depressão (HADS-D). A maioria dos participantes não apresenta sintomatologia ansiogena $(\mathrm{n}=76)$ nem depressiva $(\mathrm{n}=74)$.

Em relação à LSNS-6, observou-se uma pontuação total de $14,67 \pm 4,55$, e uma pontuação média de 8,55 $\pm 2,95$ para o domínio "família" e de 6,12 \pm 3,48 para o domínio "amigos". Considerando as pontuações totais da LSNS-6, observou-se que a maioria dos participantes $(\mathrm{n}=74)$ não se encontra em risco de isolamento social porque apresenta uma pontuação global média igual ou superior a 12 .

Relativamente às variáveis de condição física, concretamente o equilíbrio funcional, avaliado pelo TSL5 ${ }^{\star}$ verificou-se um tempo médio em segundos de 12,89 $\pm 5,37$. Na mobilidade funcional, avaliada pelo TUG verificou-se um tempo médio em segundos de 10,96 $\pm 5,16$.

\section{Preditores do medo de cair - regressão linear}

A análise do modelo de regressão linear permite observar a existência de um declive negativo e estatisticamente significativo entre a pontuação na CEA e o gênero feminino, ou seja, as mulheres terão em média menos 4,22 pontos na CEA do que os homens; no caso da idade, à medida que a idade dos participantes aumenta, a pontuação na 
Tabela 1. Características sociodemográficas e dados gerais de avaliação.

\begin{tabular}{llr}
\hline & \multicolumn{1}{c}{ Variáveis } & n (\%) \\
\hline Gênero & Feminino & $56(57,1)$ \\
& Masculino & $42(42,9)$ \\
Idade $\left(\mathrm{M} \pm \mathrm{DP}^{*}\right)$ & $74,07 \pm 8,74$ & \\
Escolaridade $(\mathrm{M} \pm \mathrm{DP})$ & $3,68 \pm 2,25$ & $10(10,2)$ \\
& 0 anos & $76(77,6)$ \\
& $1-4$ anos & $12(12,2)$ \\
& mais de 4 anos & \\
Estado Civil & & $9(9,2)$ \\
& Solteiro & $72(73,5)$ \\
& Casado & $1(1,0)$ \\
& Divorciado & $16(16,3)$ \\
& Viúvo & $12(12,2)$ \\
& & $4(4,1)$ \\
Ocupação Habitual & Emprego remunerado & $1(1,0)$
\end{tabular}

Quedas

Não

Medo de cair

Sim

Percepção da saúde física

Muito boa
Boa
Moderada
Má

Percepção da saúde mental e emocional

Muito boa
Boa
Moderada
Má
$85,93 \pm 17,29$

$\mathrm{CEA}(\mathrm{M} \pm \mathrm{DP})$

$85,93 \pm 17,29$

\begin{tabular}{llr} 
HADS-A $(\mathrm{M} \pm \mathrm{DP})$ & $4,81 \pm 5,56$ & \\
& Normal & $76(77,6)$ \\
& Ligeira & $4(4,1)$ \\
& Moderada & $11(11,2)$ \\
& Severa & $7(7,1)$ \\
& $4,87 \pm 5,71$ & \\
HADS-D $(\mathrm{M} \pm \mathrm{DP})$ & Normal & $74(75,5)$ \\
& Ligeira & $6(6,1)$ \\
& Moderada & $10(10,2)$ \\
& Severa & $8(8,2)$ \\
& & $8,55 \pm 2,95$ \\
LSNS-6 & & $6,12 \pm 3,48$ \\
Domínio “Família” $(\mathrm{M} \pm \mathrm{DP})$ & & $12,89 \pm 5,37$ \\
Domínio “Amigos" $(\mathrm{M} \pm \mathrm{DP})$ & & $10,96 \pm 5,16$ \\
TSL5 & & \\
TUG $(\mathrm{M} \pm \mathrm{DP})$ & & \\
\hline
\end{tabular}

$80(81,6)$

$18(18,4)$

$48(49,0)$

$50(51,0)$

CEA diminui, contudo não é um resultado estatisticamente significativo (Tabela 2).

Em relação à escolaridade, no caso dos participantes sem educação formal como o declive é negativo, significa que haverá um decréscimo na pontuação total da escala, contudo os resultados não são significativos.

$\mathrm{Na}$ questão “Tem medo de cair?", não ter medo de cair significa um aumento de 10,08 pontos na CEA, um resultado estatisticamente significativo.

Na percepção de saúde física, o declive em todas as categorias é positivo, e a pontuação mais alta na escala é obtida na categoria "boa".

Já a percepção de saúde mental e emocional dos participantes não afeta significativamente o valor da pontuação total da escala, apesar do declive nas categorias "muito boa" e "boa" ser positivo, ou seja, nestas categorias a pontuação na escala aumentaria, quando comparada com a categoria "moderada" em que a pontuação da escala diminuiria.

Verificou-se que a presença de sintomatologia depressiva e uma pontuação mais elevada no TSL5* emergem como preditores de medo de cair avaliado pela CEA. Já os estados de sintomatologia ansiógena, o isolamento social (LSNS-6) e a mobilidade funcional (TUG) não afetam significativamente a pontuação global da CEA.

$12(12,2)$

$30(30,6)$

$42(42,9)$

$14(14,3)$

$16(16,3)$

$49(50,0)$

$25(25,5)$

$8(8,2)$

\section{Discussão}

Este estudo procurou determinar os preditores de medo de cair em pessoas idosas portuguesas na comunidade.

Verificou-se que as mulheres têm em média menos 4,22 pontos na CEA do que os homens, ou seja, o gênero feminino é preditor do medo de cair; no caso da idade à medida que a idade dos participantes aumenta, a pontuação na CEA diminui, contudo não é um resultado estatisticamente significativo. Este resultado corrobora os estudos de Lach ${ }^{16}$, Zijlstra et al. ${ }^{17}$, Scheffer et al. ${ }^{5}$, Denkinger et al. ${ }^{40}$ no que respeita à prevalência do medo de cair aumentar com o gênero feminino, já a idade e a escolaridade não emergiram como variáveis preditoras do medo de cair, como concluíram os mesmos autores.

Segundo a Organização Mundial de Saúde ${ }^{3}$ as pessoas com mais de 70 anos, principalmente do gênero feminino, apresentam taxas de mortalidade associadas a quedas superiores às pessoas mais jovens. As causas para este fenômeno podem estar associadas à diminuição da força muscular ${ }^{9}$, à 
Tabela 2. Resultados do Modelo de Regressão Linear.

\begin{tabular}{|c|c|c|c|}
\hline Variáveis & Coeficientes & p-value & IC (95\%) \\
\hline Gênero & $-4,22$ & 0,01 & {$[-7,58 ;-0,86]$} \\
\hline \multicolumn{4}{|l|}{ Idade } \\
\hline 60-69 anos & 0,38 & 0,95 & {$[-10,45 ; 11,22]$} \\
\hline 70-79 anos & $-0,09$ & 0,99 & {$[-10,52 ; 10,33]$} \\
\hline $80-89$ anos & $-0,71$ & 0,88 & {$[-10,19 ; 8,77]$} \\
\hline \multicolumn{4}{|l|}{ Educação } \\
\hline 0 anos & $-5,38$ & 0,07 & {$[-13,37 ; 0,60]$} \\
\hline 1 a 4 anos & $-2,72$ & 0,13 & {$[-8,57 ; 1,10]$} \\
\hline Medo de Cair & 10,08 & 0,00 & {$[6,07 ; 14,08]$} \\
\hline \multicolumn{4}{|c|}{ Percepção de Saúde Física } \\
\hline Muito boa & 2,98 & 0,46 & {$[-4,86 ; 10,81]$} \\
\hline Boa & 7,27 & 0,01 & {$[1,47 ; 13,08]$} \\
\hline Moderada & 6,56 & 0,02 & {$[1,23 ; 11,90]$} \\
\hline \multicolumn{4}{|c|}{ Percepção de Saúde Mental e Emocional } \\
\hline Muito boa & 4,25 & 0,32 & {$[-4,08 ; 12,57]$} \\
\hline Boa & 2,44 & 0,50 & {$[-4,56 ; 12,57]$} \\
\hline Moderada & $-2,37$ & 0,50 & {$[-9,25 ; 4,51]$} \\
\hline \multicolumn{4}{|l|}{ HADS-A } \\
\hline normal & $-16,80$ & 0,05 & {$[-33,75 ; 0,15]$} \\
\hline ligeira & $-13,77$ & 0,08 & {$[-29,10 ; 1,56]$} \\
\hline moderada & $-5,14$ & 0,25 & {$[-13,85 ; 3,57]$} \\
\hline \multicolumn{4}{|l|}{ HADS-D } \\
\hline normal & 26,04 & 0,00 & {$[9,48 ; 42,61]$} \\
\hline ligeira & 18,54 & 0,02 & {$[3,28 ; 33,80]$} \\
\hline moderada & 15,19 & 0,00 & {$[6,78 ; 23,59]$} \\
\hline LSNS-6 & 0,01 & 0,99 & {$[-4,63 ; 4,65]$} \\
\hline TSL5 $^{*}$ & $-1,52$ & 0,00 & {$[-2,17 ;-0,87]$} \\
\hline TUG & $-0,11$ & 0,75 & {$[-0,79 ; 0,57]$} \\
\hline
\end{tabular}

osteoporose, à prevalência de doenças crônicas, a uma maior restrição à vida doméstica nas mulheres $^{10}$, e a deficits nas funções sensoriais que afetam o equilíbrio e a marcha na realização de tarefas múltiplas ${ }^{41}$. Estas causas poderão ser minimizadas através da avaliação do risco individual e doméstico, sobretudo a utilização de precauções básicas de segurança durante as atividades de vida diária, como uma boa iluminação, utilização de calçado adequado e adaptações ambientais ${ }^{41-43}$.

Com o processo de envelhecimento podem ainda ocorrer alterações vertibulares que interferem no equilíbrio gerando tonturas ${ }^{44}$. Estas são o principal fator associado a quedas nas pessoas idosas ${ }^{45}$ potenciando medo de cair ${ }^{46}$. Segundo Ricci et al. há uma maior prevalência de tonturas nas pessoas idosas com histórico de quedas quando comparados aos que não apresentam tal histórico ${ }^{45}$.

O risco de queda encontra-se também associado à baixa escolaridade ${ }^{47}$, uma vez que baixa escolaridade pode ser indicativa de um menor conhecimento de meios de prevenção. No caso específico da CEA, existem evidências que a versão original desta escala apresenta uma maior capacidade de resposta para pessoas idosas com nível de funcionalidade mais elevado e abrange atividades com grau de dificuldade acrescido ${ }^{14,21}$, e neste estudo a maioria dos participantes tem baixa escolaridade, razão pela qual a escolaridade pode não ter surgido como preditora do medo de cair.

Os presentes resultados vão também ao encontro de estudos prévios no que respeita à relação entre o medo de cair avaliado pela CEA e as variáveis de saúde, nomeadamente a questão “Tem medo de cair? ” e a percepção de saúde. O medo de cair é um determinante de quedas relacionado com fatores pessoais que pode levar à restrição da atividade e participação, aumentando a perda da confiança e diminuindo a capacidade para evitar as quedas ${ }^{11,13,20,23,48}$.

Importa também referir que uma pior percepção de saúde emergiu como preditor do medo de cair, resultado que se encontra em conformidade com os estudos de Lach $^{16}$, Zijlstra et al. ${ }^{17}$, Scheffer et al. ${ }^{5}$, nos quais se observou que o medo de cair nas pessoas idosas representa uma estreita relação com a fraca percepção do estado de saúde e autoeficácia, indicativo de menor condição física ${ }^{40}$, e declínios na motivação e confiança, com implicações diretas na adesão às intervenções preventivas de quedas.

Analisando especificamente a pontuação na CEA com a HADS, verificou-se que a depressão emergiu como preditor do medo de cair, ou seja, na presença de sintomatologia depressiva, a pontuação na CEA é menor, indicativa de maior medo de cair. Este resultado está em linha com estudos anteriores nos quais se observou uma associação entre o medo de cair e os estados de depressão $0^{4,5,15,17,19,20,22}$, ou seja, os sintomas depressivos tendem a condicionar a participação em atividades físicas e funcionais por medo da incapacidade em realizá-las, enfatizando a necessidade de estratégias de intervenção multidimensionais para minimizar o medo de cair e, consequente, o risco de queda. Já a ansiedade não surgiu como fator preditor do medo de cair, no entanto tem influência direta na marcha e no equilíbrio funcional, afeta a autoeficácia durante a realização das AVD e consequentemente aumenta o medo de cair ${ }^{40}$.

A LSNS-6 apresentou uma pontuação média superior para o domínio "família" $(8,55 \pm 2,95)$ e verificou-se que a maioria dos participantes $(\mathrm{n}=$ 74) não se encontra em risco de isolamento so- 
$\mathrm{cial}^{27}$. No entanto, apesar de não ser um resultado significativo, verificou-se que os participantes sem risco de isolamento social apresentam em média uma pontuação mais elevada na CEA, ou seja, existe uma relação negativa e significativa entre o medo de cair e o apoio social recebido, resultado em conformidade com estudos prévios ${ }^{40}$. Razões culturais poderão explicar o fato deste resultado ser pouco significativo e neste estudo o isolamento social não ser um preditor forte do medo de cair, na medida em que a sociedade portuguesa se caracteriza ainda por fortes laços de solidariedade familiar e comunitária ${ }^{49} \mathrm{em}$ particular nos meios maioritariamente rurais, onde o estudo foi conduzido. Neste sentido, as redes de entreajuda baseadas em laços de parentesco e de vizinhança característicos do meio rural, através das quais pequenos grupos sociais trocam apoio numa lógica de reciprocidade poderá ajudar a compreender este resultado.

Relativamente às variáveis de condição física, concretamente o equilíbrio funcional, avaliado pelo TSL5 $^{\star}$ verificou-se um tempo médio em segundos de $12,89 \pm 5,37$, e na mobilidade funcional, avaliada pelo TUG verificou-se um tempo médio em segundos de 10,96 $\pm 5,16$, pontuação superior ao que a literatura considera como um desempenho normal, independente e sem alterações no equilíbrio (10 segundos) ${ }^{28}$.

Analisando de que forma a pontuação no TSL5* e no TUG predizem a pontuação na CEA, verificou-se que, à medida que o tempo médio aumenta no TSL5* e TUG, a pontuação CEA é menor, indicativo de maior medo de cair. Contudo neste estudo o resultado apenas foi significativo no TSL5 ${ }^{\star}$. De fato, a prevalência do medo de cair nas pessoas idosas representa uma estreita relação com múltiplos fatores, nomeadamente a falta de equilíbrio, decréscimo na mobilidade e marcha $^{5,20,40}$.

O presente estudo apresenta algumas limitações. Utiliza uma amostra por conveniência, selecionada a partir da mesma zona geográfica, comprometendo a generalização dos resultados à população idosa portuguesa da comunidade. Para além disso, os questionários foram aplicados em contexto de entrevista e não por autopreenchimento, podendo levar a algum viés nas respostas, particularmente daqueles com baixo grau de escolaridade. Em estudos futuros seria desejável utilizar uma amostra probabilística mais alargada e analisar o constructo medo de cair com recurso a outros instrumentos de avaliação para determinar se os resultados se mantinham idênticos aos alcançados pela CEA.

Apesar das limitações, os resultados do presente estudo enfatizam a necessidade do desenvolvimento de estratégias de intervenção multifatoriais e costumizadas com vista à prevenção de quedas e ao medo a elas associado. Neste sentido, são necessários estudos que analisem como é que as intervenções poderão afetar o medo de cair e a sua associação com o gênero, percepção de saúde e variáveis psicológicas.

\section{Colaboradores}

SCA Santos trabalhou na análise e interpretação dos dados e na sua redação, e DMP Figueiredo trabalhou na concepção do projeto e na revisão crítica. 


\section{Referências}

1. Lamb SE, Jorstad-Stein EC, Hauer K, Becker C. Development of a common outcome data set for fall injury prevention trials: the Prevention of Falls Network Europe consensus. J Am Geriatr Soc 2005; 53(9):16181622.

2. Sethi D, Racioppi F, Baumgarten I, Vida P. Injuries and violence in Europe: why they matter and what can be done. Copenhagen: World Health Organization; 2006. [acessado 2016 Jan 20]. Disponível em: http:// www.euro.who.int/_data/assets/pdf_file/0005/98762/ E88037.pdf

3. World Health Organization (WHO). Media center. Falls; 2012. [acessado 2016 Jan 20]. Disponível em: http://www.who.int/mediacentre/factsheets/fs344/en/

4. Carvalho J, Pinto J, Mota J. Actividade física, equilíbrio e medo de cair. Rev Portuguesa das Ciências do Desporto 2007; 7(2):225-231.

5. Scheffer AC, Schuurmans MJ, van Dijk N, van der Hooft T, Rooij SE. Fear of falling: measurement strategy, prevalence, risk factors and consequences among older persons. Age Ageing 2008; 37(1):19-24.

6. Rubenstein LZ. Falls in older people: Epidemiology, risk factors and strategies for prevention. Age Ageing 2006; 35(2):37-41.

7. National Institute for Health and Care Excellence. Falls - Assessment and prevention of falls in older people. Manchester: National Institute for Health and Care Excellence; 2013. [acessado 2016 Jan 20]. Disponível em: https://www.nice.org.uk/guidance/cg161

8. Stalenhoef PA, Diederiks JP, Knottnerus JA, Kester AD, Crebolder HF. A risk model for the prediction of recurrent falls in community dwelling elderly: A prospective cohort study. J Clin Epidemiol 2002; 55(11):1088-1094.

9. Stevens JA, Sogolow ED. Gender differences for non-fatal unintentional fall related injuries among older adults. Inj Prev 2005; 11(2):115-119.

10. Perracini MR., Ramos LR. Fall-related factores in cohort of elderly community residents. Rev Saude Publica 2002; 36(6):709-716.

11. Lord S, Sherrington C, Menz H, Close J. Falls in Older People: Risk Factors and Strategies for Prevention. $2^{\text {nd }}$ ed. Cambridge: University of Cambridge; 2007.

12. Tinetti ME, Kumar C. The patient who falls: "It's allways a trade-off". JAMA 2010; 303(3):258-266.

13. Todd C, Skelton D. What are the Main Risk Factors for Falls Amongst Older People and what are the Most Effective Interventions to Prevent These Falls? Copenhagen: World Health Organization; 2004. [acessado 2016 Jan 20]. Disponível em: http://www.euro.who.int/_data/ assets/pdf_file/0018/74700/E82552.pdf

14. Legters K. Fear of Falling. Physical Therapy 2002; 82(3):264-272.

15. Gai J, Gomes L, Jansen de Cárdenas C. Ptofobia: O Medo de Cair em Pessoas Idosas. Acta Médica Portuguesa 2008; 22(1):83-88.

16. Lach HW. Incidence and risk factors for developing fear of falling in older adults. Public Health Nursing 2005; 22(1):45-52.

17. Zijlstra GAR, van Haastregt JCM, van Eijk JTM, van Rossum E, Stalenhoef PA, Kempen GIJM. Prevalence and correlates of fear of falling, and associated avoidance of activity in the general population of community-living older people. Age Ageing 2007; 36(3):304-309.
18. Tinetti ME, Powell L. Fear of Falling and Low Self-efficacy: A Cause of Dependence in Elderly Persons. $J$ Gerontol 1993; 48(n. esp.):35-38.

19. Hadjistavropoulos T, Delbaere K, Fitzgerald TD. Reconceptualizing the role of fear of falling and balance confidence in fall risk. J Aging Health 2011; 23(1):3-23.

20. Reelick MF, Van Iersel MB, Kessels RP, Rikkert M. The influence of fear of falling on gait and balance in older people. Age Ageing 2009; 38(4):435-440.

21. Moore DS, Ellis R. Measurement of fall-related psychological constructs among independent-living older adults: A review of the research literature. Aging Mental Health 2008; 12(6):684-699.

22. Jahana KO, Diogo MJDE. Quedas em idosos principais causas e consequências. Cien Saude Colet 2007; 4(17):148-153.

23. Ribeiro AP, Souza ER, Atie S, Souza A, Schilithz AO. A influência das quedas na qualidade de vida de idosos. Cien Saude Colet 2008; 13(4):1265-1273.

24. Branco PS. Validação da Versão Portuguesa da Activities-specific Balance Confidence Scale. Rev Sociedade Portuguesa Medicina Física e Reabilitação 2010; 19(1):20-25.

25. Bryman A, Cramer D. Quantitative data analysis with IBM SPSS Statistics 17, 18 and 19: A guide for social scientists. New York: Routledge; 2011.

26. Pais-Ribeiro J, Silva I, Ferreira T, Martins A, Meneses R, Baltar M. Validation study of a Portuguese version of the Hospital Anxiety and Depression Scale. Psychol, Health Med 2007; 12(2):225-237.

27. Ribeiro O, Teixeira L, Duarte N, Azevedo MJ, Araújo L, Barbosa S, Paúl C. Versão Portuguesa da Escala Breve de Redes Sociais de Lubben (LSNS-6). Rev Kairós de Gerontologia 2012; 15(1):217-234.

28. Podsiadlo D, Richardson S. The Timed Up \& Go: A test of basic functional mobility for frail elderly persons. $J$ Am Geriatr Soc 1991; 39(2):142-148.

29. Bohannon R. Reference Values for the Five-Repetition Sit-To-Stand Test: A Descriptive Meta-Analysis of Data From Elders. Perceptual Motor Skills 2006; 103(1):215222.

30. World Health Organization. ICF Checklist. Geneva: World Health Organization; 2003 [acessado 2016 Jan 20]. Disponível em: http://www.who.int/classifications /icf/training/icfchecklist.pdf

31. Powell LE, Myers AM. The Activities-specific Balance Confidence (ABC) Scale. J Gerontol A Biol Sci Med Sci 1995; 50(1):28-34.

32. Huang TT, Wang WS. Comparison of three established measures of fear of falling in community-dwelling older adults: psychometric testing. Int J Nurs Stud 2009; 46(10):1313-1319.

33. Ansai J, Glisoi S, Oliveira T, Soares A, Cabral K, Sera C, Paschoal S. Revisão de dois instrumentos clínicos de avaliação para predizer risco de quedas em idosos. Rev Bras Geriatria e Gerontologia 2014; 17(1):177-189.

34. Greene BR, O'Donovan A, Romero-Ortuno R, Cogan L, Scanaill CN, Kenny RA. Quantitative falls risk assessment using the timed up and go test. IEEE Trans Biomed Eng 2010; 57(2):2918-2926. 
35. Goldberg A. The five-times-sit-to-stand-test (FTSST), the short version of the activities-specific balance confidence $(\mathrm{ABC})$ scale, and fear of falling predict step execution time (SET) in older adults. Arch Gerontol Geriatr 2012; 54(3):434-438.

36. Annweiler C, Schott a-M, Abellan van Kan G, Rolland Y, Blain H, Fantino B, Beauchet O. The Five-Times-Sitto-Stand test, a marker of global cognitive functioning among community-dwelling older women. J Nutr Health Aging 2011; 15(4):271-276.

37. Whitney SL, Wrisley DM, Marchetti GF, Gee MA, Redfern MS, Furman JM. Clinical Measurement of Sit-toStand Performance in People With Balance Disorders: Validity of Data for the Five-Times-Sit-to-Stand Test. Physical Therapy 2005; 85(10):1034-1045.

38. Schaubert KL, Bohannon RW. Reliability and validity of three strength measures obtained from community-dwelling elderly persons. J Strength Conditioning Research 2005; 19(3):717-720.

39. Tiedemann A, Shimada H, Sherrington C, Murray S, Lord S. The comparative ability of eight functional mobility tests for predicting falls in community-dwelling older people. Age Ageing 2008; 37(4):430-435.

40. Denkinger MD, Lukas A, Nikolaus T, Hauer K. Factors Associated with Fear of Falling and Associated Activity Restriction in Community-Dwelling Older Adults: A Systematic Review. Am J Geriatr Psychiatry 2015; 23(1):73-85.

41. Pohl P, Ahlgren C, Nordin E, Lundquist A, Lundin-Olsson L. Gender perspective on fear of falling using the classification of functioning as the model. Disabil Rehabil 2015; 37(3):214-222.

42. Santos JS, Valente JM, Carvalho MA, Galvão KM, Kasse CA. Identificação dos fatores de riscos de quedas em idosos e sua prevenção. Rev Equilíbrio Corporal Saúde 2013; 5(2):53-59.

43. Albert SM, Edelstein O, King J, Flatt J, Lin CJ, Boudreau R, Newman AB. Assessing the Quality of a Non-randomized Pragmatic Trial for Primary Prevention of Falls among Older Adults. Prevention Science 2015; 16(1):31-40.
44. Duarte GA, Soldera CLC. Association among complaint of dizziness, fear of falling and prior occurrence of falls in elderly people. Geriatr Gerontology Aging 2013; 7(3):209-214.

45. Ricci NA, Gonçalves DFF, Coimbra IB, Coimbra AMV. Fatores associados ao histórico de quedas de idosos assistidos pelo Programa de Saúde da Família. Saúde Soc 2010; 19(4):898-909.

46. Gananca FF, Gazzola JM, Aratani MC, Perracini MR, Gananca MM. Circunstâncias e consequências de quedas em idosos com vestibulopatia crónica. Rev Bras Otorrinolaringol 2006; 72(3):388-393.

47. World Health Organization (WHO). Global report on falls prevention in older age. Geneva: WHO; 2007. [acessado 2016 Jan 20]. Disponível em: http://www.who.int/ ageing/publications/Falls_prevention7March.pdf

48. Deshpande N, Metter JE, Lauretani F, Bandinelli S, Ferrucci L. Interpreting Fear of Falling in the Elderly: What Do We Need to Consider? J Geriatr Phys Ther 2009; 32(3):91-96.

49. Pimentel L. O lugar do idoso na família. Contextos e trajectórias. Coimbra: Quarteto Editora; 2001.

Artigo apresentado em 29/02/2016

Aprovado em 16/03/2017

Versão final apresentada em 18/03/2017 